\title{
Malignant Subcapsular Adrenal Tumor
}

National Cancer Institute

\section{Source}

National Cancer Institute. Malignant Subcapsular Adrenal Tumor. NCI Thesaurus. Code C83489.

A metastasizing tumor located underneath the adrenal capsule. 